\title{
Body Composition Predicts Survival in Patients with Hepatocellular Carcinoma Treated with Transarterial Chemoembolization
}

Neehar D. Parikh, MD, MS ${ }^{1,2}$
Peng Zhang, PhD',4
Amit G. Singal, MD, MS
Brian A. Derstine, PhD
Venkat Krishnamurthy, MD²
Pranab Barman, MD'
Akbar K. Waljee, MD, MSc',2,
Grace L. Su, MD

${ }^{1}$ Division of Gastroenterology, University of Michigan Medical School, Ann Arbor, MI, ${ }^{2} V A$ Ann Arbor Healthcare System, Ann Arbor, MI, ${ }^{3}$ Department of Surgery, University of Michigan Medical School, Ann Arbor, MI, ${ }^{4}$ Morphomic Analysis Group, University of Michigan Medical School, Ann Arbor, MI, ${ }^{5}$ Division of Digestive and Liver Diseases, UT Southwestern Medical Center, Dallas, TX, ${ }^{6}$ Veterans Affairs Center for Clinical Management Research, VA Ann Arbor Healthcare System, Ann Arbor, MI, USA
Correspondence: Grace L. Su, MD

VA Ann Arbor Healthcare System,

2215 Fuller Road, Ann Arbor, MI 48105, USA

Tel: $1-7348455865$

Fax: 1-7349367392

E-mail: gsu@umich.edu

Received March 31, 2017

Accepted May 24, 2017

Published Online June 1, 2017

${ }^{*}$ Neehar D. Parikh and Peng Zhang contributed equally to this work.

\section{Purpose}

The prognosis of patients with hepatocellular carcinoma (HCC) undergoing transarterial chemoembolization (TACE) is often uncertain. We aimed to utilize analytic morphomics, a high-throughput imaging analysis, to assess if body composition is predictive of post-TACE survival.

\section{Materials and Methods}

We included patients from a single center (Ann Arbor VA) who had TACE as the primary treatment for HCC and had a pre-treatment computed tomography scans. Univariate analysis and multivariate conditional inference tree analysis were utilized to identify the morphomic characteristics predictive of 1-year survival. Results were validated in an external cohort (University of Michigan Health System) of HCC patients who underwent TACE as their primary treatment.

\section{Results}

In the 75 patients in the derivation cohort, median survival was 439 (interquartile range, 377 to 685 ) days from receipt of TACE, with 1-year survival of $61 \%$. Visceral fat density (VFD) was the only morphomic factor predictive of overall and 1-year survival $(p<0.001)$. Patients with VFD above the 56th percentile had a 1-year survival of $39 \%$ versus $78 \%$ for those below the 56th percentile. VFD also correlated with 1-year survival in the external validation cohort (44\% vs. $72 \%, p<0.001$ ). In a secondary analysis, patients with higher VFD were significantly more likely to experience hepatic decompensation after TACE $(p<0.001)$.

\section{Conclusion}

VFD served as an objective predictor of mortality in patients undergoing TACE, possibly through its ability to predict hepatic decompensation. VFD may serve as a radiographic biomarker in predicting TACE outcomes.

\section{Key words}

Hepatocellular carcinoma, Chemoembolization, Therapeutic 


\section{Introduction}

Hepatocellular carcinoma (HCC) is increasing in frequency and is a leading cause of cancer-related mortality worldwide [1]. While tumor biology heterogeneity is an important determinant of outcome, patient-level factors are clearly important determinants of outcome. Established prognostic factors at diagnosis include not only tumor burden, but also degree of liver dysfunction, and the patient's functional status. The Barcelona Clinic Liver Cancer (BCLC) system incorporates each of these variables and is the most widely used staging system in the Western hemisphere to assess prognosis in patients with HCC [2]. While societal guidelines use the BCLC to provide guidance for initial treatment decisions, its ability to predict treatment response and outcomes such as long-term survival, particularly in the setting of HCCdirected therapy, is currently limited [3]. Furthermore, we have previously found that community practices often deviated from practice guidelines especially in regards to the utilization of transarterial chemoembolization (TACE) [4]. An ability to objectively risk-stratify patients could result in more judicious use of therapy or improved monitoring protocols for those patients at highest risk for treatment-related adverse events.

Given high rates of late stage tumor detection, most HCC patients are not eligible for or have limited access to curative therapies such as surgical resection, liver transplantation, or radiofrequency ablation [5]. Therefore, locoregional therapies including TACE are the most widely used treatments for HCC patients in clinical practice. While there are some clear contraindications to TACE, we have limited ability to determine a priori who will experience the largest survival benefit with treatment. Survival after TACE is impacted by multiple factors, including treatment response and patient tolerance, most notably development of new or worsening liver dysfunction or decompensation [4]. While functional status has been shown to be predictive of outcomes in patients with HCC, the Eastern Cooperative Oncology Group (ECOG) score may have less prognostic value in the subset of patients undergoing TACE $[3,4]$. This may be related to measurement bias, given its subjective nature and inter-observer variability $[6,7]$. A potential alternative approach to quantify functional status is to measure a patient's body composition, including muscle, fat, and bone characteristics. This can be done via computed tomography (CT) scan which is particularly relevant as patients with HCC universally have baseline imaging as part of diagnosis and staging.

Analytic morphomics is a novel approach that uses highthroughput semi-automated image-processing techniques to assess body composition [8,9]. The methodology analyzes pre-existing $\mathrm{CT}$ scans, and precisely quantifies patient anatomical components contained within the scans. By anatomically indexing each scan, this methodology allows for objective reproducible measurements which can be utilized to assess the heterogeneity of patients. We have previously used analytic morphomics to identify predictors for cirrhosis development, surgical outcomes, liver transplantation, and patients with HCC [9-12].

The primary aim of this study was to determine if analytic morphomic characteristics could independently predict survival among patients undergoing TACE. A secondary aim was to determine whether analytic morphomics characteristics were able to predict hepatic decompensation after TACE.

\section{Materials and Methods}

\section{Study populations}

Our derivation cohort (Ann Arbor VA [AAVA] Cohort) consisted of all patients with a new diagnosis of HCC who underwent TACE as primary treatment at the Veterans Administration Ann Arbor Healthcare System (VAAAHS) between January 2006 and December $2012(n=109)$. The VAAAHS is a tertiary care VA hospital system in the upper midwest of the United States. Patients were identified using multidisciplinary liver tumor conference presentation lists and administrative databases using International Classification of Diseases, 9th edition (ICD-9) codes (155.0 and 155.2), as previously described [4]. We excluded patients without pre-treatment CT scans $(\mathrm{n}=27)$, patients who subsequently underwent curative therapy such as liver transplantation $(\mathrm{n}=2)$, and those without complete morphomics data at T11 $(\mathrm{n}=5)$.

The validation cohorts consisted of all patients with treatment naïve HCC who underwent TACE at the University of Michigan (UMHS cohort) between January 2005 and March 2012 [13]. The University of Michigan is a tertiary care transplant center, with a wide catchment area in the upper midwest United States. Patients in the validation cohort were identified using ICD-9 codes for HCC, tumor conference presentation lists, and HCC databases as previously described $[14,15]$. We applied the same exclusion criteria for the UMHS cohort as the AAVA cohort.

HCC cases in both cohorts were adjudicated to confirm they met diagnostic criteria based on American Association for the Study of Liver Diseases guidelines [16]. For tumors larger than $1 \mathrm{~cm}, \mathrm{HCC}$ diagnosis required a typical vascular pattern on dynamic imaging (arterial enhancement and delayed washout) or histology. Patients in both cohorts were discussed in multidisciplinary Liver Tumor Boards for man- 
agement decisions, and curative treatments (liver transplantation, resection, and local ablation) were recommended for patients with early stage HCC whenever possible. Therefore, TACE was primarily used for patients in whom curative therapies were not applicable. This study was approved by the Institutional Review Boards at the AAVA Healthcare System and the UMHS.

\section{Analytic morphomics}

Pre-treatment CT studies were analyzed using analytic morphomics in order to characterize patient body composition, as previously described [8,17-19]. Analytic morphomics is a platform of semi-automatic image analysis software which is able to anatomically index CT scan data so that each measurement is precise and reproducible. Briefly, coded de-identified DICOM (Digital Imaging and Communications in Medicine) files of all CT scans were loaded into the analytic morphomics server. DICOM files are the standard underlying data files that are used to store all CT image data. Given the method of processing and required data, both contrast and non-contrast scans could be used. A semi-automated high throughput methodology with algorithms programmed in MATLAB (MathWorks Inc., Natick, MA) enabled image processing and analysis. All algorithms involved a combination of user-defined points, automated image processing, and user editing and verification. All imaging studies were first anatomically indexed using semiautomated identification of spinal vertebral levels to allow for accurate and standardized measurements of the same area in each patient. A single slice was chosen, as this would include all required body composition features while minimizing processing time and potential radiation exposure for future prospective studies. For this study, we examined morphomic features that were measured at the inferior aspect of the 11th thoracic vertebral level. This anatomic landmark was chosen because this was felt to have the highest likelihood of being available on both abdominal and chest CT scans. Guidelines recommend a staging CT chest for all patient diagnosed with HCC. All geometries were saved in a stereolithography format in the analytic morphomics database with PostgresSQL and subsequently retrieved to calculate geometric (linear distances or cross-sectional areas) and Hounsfield Unit based measurements with customized algorithms. For measurement of bone density, we used measurements of trabecular bone. For muscle mass and quality measurements, we used measurements of the dorsal muscle group which is the core muscle at the T11 anatomical level. Descriptions of all the analytic morphomic measurements used for this study can be found in the data dictionary (http://www.med.umich.edu/surgery/morphomics/data_ dictionary).
Considering that age and gender are biggest confounding factors for body compositions, all direct morphomics measurements were matched with a reference population to generate the age and sex matched percentile for each measurement. The reference population consists of over 6,000 patients who underwent CT scans primarily for trauma indications. Patients scanned for trauma, which is a relatively random event, constitutes a "normal" population to allow for referencing. All statistical analyses were based upon the percentile versions of analytic morphomics.

\section{Clinical data collection}

Patient demographics, clinical history, laboratory data and imaging results for both cohorts were obtained through review of clinical databases and electronic medical records, and extracted using standardized forms. Data regarding presence of decompensation (ascites or hepatic encephalopathy) were abstracted from clinical notes and classified as none, mild or controlled, and severe or uncontrolled. ECOG performance status, as determined by clinical provider at time of HCC presentation, was also abstracted from clinical notes. Laboratory data of interest at time of HCC diagnosis included platelet count, aspartate aminotransferase, alanine aminotransferase, bilirubin, albumin, international normalized ratio, and $\alpha$-fetoprotein. Dynamic contrast-enhanced CT or magnetic resonance imaging (MRI), as interpreted by radiologists at each site, were used to determine tumor characteristics including number of lesions, maximum tumor diameter, lymph node involvement, portal vein invasion, and presence of extrahepatic metastases. The BCLC criteria were used for tumor staging with early stage defined as BCLC stage 0 or A. Patient clinical course including dates of treatment, follow-up imaging, and death was recorded for each patient.

\section{Statistical analysis}

Post-treatment survival was estimated using Kaplan-Meier analysis. Survival was defined from time of TACE to death; survival for patients without death was censored at last clinic visit. The primary outcome for statistical analyses was the mortality risk at 1-year post-TACE given high risk of confounders influencing survival beyond 1 year. We developed a prognostic model in the AAVA Cohort by using a Cox's proportional hazard regression with forward/backward selection from a combination of analytic morphomic variables (features from Table 1) and a priori important clinical variables associated with survival (Child-Pugh class, TNM tumor burden [III and IV were combined due to limited numbers], and ECOG score). Furthermore, we employed the conditional inference tree analyses to determine thresholds 
Table 1. Summary of raw and percentile body composition features in derivation cohort

\begin{tabular}{|c|c|c|c|c|c|c|}
\hline \multirow{2}{*}{ Morphomic variable (at T11) } & \multicolumn{3}{|c|}{ Raw values } & \multicolumn{3}{|c|}{ Percentiles } \\
\hline & Median & $\overline{Q_{1}}$ & $\mathrm{Q}_{3}$ & Median & $\mathbf{Q}_{1}$ & $\mathrm{Q}_{3}$ \\
\hline Distance from the fascia to the vertebra (mm) & 160 & 146 & 173 & 62 & 39 & 80 \\
\hline Area of all muscle in the dorsal muscle group $\left(\mathrm{mm}^{2}\right)$ & 3,619 & 3,034 & 4,268 & 46 & 28 & 69 \\
\hline $\begin{array}{l}\text { Ratio of normal density muscle } \\
\text { to the dorsal muscle area (ratio) }\end{array}$ & 0.35 & 0.22 & 0.63 & 70 & 40 & 93 \\
\hline Low density dorsal muscle area $\left(\mathrm{mm}^{2}\right)$ & 938 & 662 & 1,418 & 69 & 37 & 94 \\
\hline Normal density dorsal muscle area $\left(\mathrm{mm}^{2}\right)$ & 2,625 & 2,067 & 3,245 & 37 & 21 & 57 \\
\hline Total fascia circumference (mm) & 943 & 900 & 996 & 48 & 24 & 70 \\
\hline $\begin{array}{l}\text { Eccentricity of an ellipse having the } \\
\text { same second-moments of the fascia region (ratio) }\end{array}$ & 0.62 & 0.56 & 0.65 & 29 & 12 & 47 \\
\hline Total area within the fascia region $\left(\mathrm{mm}^{2}\right)$ & 67,373 & 60,914 & 74,348 & 54 & 25 & 74 \\
\hline Subcutaneous fat area $\left(\mathrm{mm}^{2}\right)$ & 8,451 & 5,252 & 12,350 & 46 & 26 & 73 \\
\hline Bone mineral density (HU) & 180 & 153 & 226 & 50 & 29 & 79 \\
\hline Visceral fat density (HU) & -96 & -102 & -87 & 50 & 25 & 83 \\
\hline Visceral fat area $\left(\mathrm{mm}^{2}\right)$ & 8,458 & 5,553 & 11,365 & 30 & 19 & 43 \\
\hline
\end{tabular}

$\mathrm{Q}_{1}$ is the 1 st quartile and $\mathrm{Q}_{3}$ is the 3 rd quartile. The percentile value was referenced against a normal population controlling for age and gender, which are unit less. The units for the raw values were specified in the row names. The smaller eccentricity indicates a rounder body shape. HU, Hounsfield Units.

of morphomics in differentiating the risk of post-TACE mortality [20]. Unlike the traditional classification and regression tree analysis, the conditional inference tree method utilizes a formal statistical framework to address multiple comparisons between groups and avoid over-fitting. The identified thresholds of morphomic variables with the conditional inference tree analysis in the derivation AAVA cohort were further validated in the UMHS cohort. In addition, we performed subgroup analyses to determine whether analytic morphomics were predictive of survival in subpopulations of HCC patients, including (1) those patients are TACE eligible according to BCLC treatment guidelines (ECOG 0-2, no evidence of metastatic disease, Child-Pugh A or B); (2) those patients with Child-Pugh A cirrhosis; and (3) those with Child-Pugh scores of 5-7.

In a secondary analysis, we investigated whether analytic morphomics characteristics predicted development of hepatic decompensation after TACE, a strong determinant of overall survival in patients with HCC. Hepatic decompensation was defined as an increase in Child Turcotte Pugh score by $\geq 2$ points, new ascites, new encephalopathy, or an increase in total bilirubin by $\geq 2.0 \mathrm{mg} / \mathrm{dL}$ within 6 weeks of receipt of TACE and prior to receipt of any other HCCdirected therapy [21]. All statistical analyses were performed using R 3.1.0.

\section{Results}

\section{Patient population}

There were 75 patients included in the AAVA derivation cohort and 90 patients in the UMHS validation cohort. Baseline characteristics of the cohorts are shown in Table 2. The derivation cohort had a higher proportion of males, hepatitis $C$ positivity, and more advanced cancer stage when compared to the validation cohort. The vast majority of patients in both cohorts had good functional status prior to TACE (ECOG 0-1). The overall 1-year survival after TACE for both cohorts was similar-61\% for patients in the AAVA cohort and $56 \%$ in the UMHS cohort.

In the AAVA cohort, several muscle and fat characteristics differed from the age- and sex-matched reference population (Table 1). Although the overall core muscle area was near the 50th percentile, there was a predominance of low density muscle, suggesting more fatty infiltration of muscle in HCC patients. The total area within fascia region, subcutaneous fat area and fascia circumference was similar to the reference population. However, the visceral fat area was significantly lower in the TACE population ( $\mathrm{p}<0.001)$. Also, the eccentricity of fascia was also significantly smaller in the TACE population $(p<0.001)$, suggesting a more-round abdominal shape of these patients. S1 Table shows the body composition 
Table 2. Clinical descriptive data of derivation and validation cohorts

\begin{tabular}{|c|c|c|c|}
\hline & Derivation cohort $(n=75)$ & Validation cohort $(\mathrm{n}=90)$ & p-value \\
\hline Age (yr) & $60.0(56-62)$ & $59.0(54-65)$ & 0.38 \\
\hline Male sex & $73(97)$ & $76(84)$ & 0.09 \\
\hline BMI & $27.7(23.8-31.1)$ & $29.1(25.7-32.5)$ & 0.46 \\
\hline Diabetes & $25(25)$ & $32(36)$ & 0.18 \\
\hline Hypertension & $51(68)$ & $53(59)$ & 0.26 \\
\hline $\mathrm{HCV}+$ & $63(84)$ & $55(61)$ & $<0.001$ \\
\hline Child-Pugh class A/B / C & $47 / 24 / 4$ & $50 / 31 / 9$ & 0.49 \\
\hline TNM stage I/II/ III / IV & $36 / 25 / 13 / 1$ & $23 / 30 / 23 / 14$ & $<0.001$ \\
\hline Barcelona stage $0 / \mathrm{A} / \mathrm{B} / \mathrm{C} / \mathrm{D}$ & $1 / 16 / 15 / 38 / 5$ & $2 / 23 / 32 / 24 / 9$ & 0.02 \\
\hline ECOG status $0 / 1 / 2 / 3$ & $35 / 35 / 4 / 1$ & $58 / 26 / 4 / 2$ & 0.09 \\
\hline $\mathrm{AFP}$ & $38.5(13.1-215.4)$ & $29.4(7.9-235.3)$ & 0.46 \\
\hline Total bilirubin & $1.2(0.7-1.9)$ & $1.2(0.7-2.0)$ & 0.93 \\
\hline Albumin & $3.2(2.8-3.8)$ & $3.6(2.1-3.98)$ & 0.01 \\
\hline MELD & $9(8-11)$ & $10(7-12)$ & 0.47 \\
\hline Multifocal & $40(53)$ & $55(61)$ & 0.35 \\
\hline
\end{tabular}

Values are presented as median (range) or number (\%). For continuous variables, median and interquartile were shown, and p-values were from Mann-Whitney $U$ test. For categorical variables, counts (and frequency if binary) were shown, and p-values were from Fisher exact test. BMI, body mass index; HCV, hepatitis C virus; ECOG, Eastern Cooperative Oncology Group; AFP, $\alpha$-fetoprotein; MELD, Mayo End Stage Liver Disease score.

Table 3. Cox proportional hazard model for survival after TACE

\begin{tabular}{lcc} 
& HR & 95\% Confidence interval \\
Child-Pugh class B (reference: class A) & 2.15 & $1.03-4.48$ \\
Child-Pugh class C (reference: class A) & 2.69 & $0.82-8.80$ \\
TNM stage II (reference stage I) & 1.88 & $0.95-3.72$ \\
TNM stage III or IV (reference: stage I) & 2.56 & $1.13-5.80$ \\
Visceral fat density percentile & 1.01 & $1.00-1.03$ \\
\hline
\end{tabular}

TACE, transarterial chemoembolization; HR, hazard ratio.

characteristics and percentiles of the validation cohort. While patients in the validation cohort had similar muscle characteristics as the AAVA cohort, they had a larger fascia compartment and higher visceral fat density. Comparison of the morphomic characteristics between the derivation and validation cohorts is shown in S2 Table.

\section{Post-TACE survival}

In the derivation AAVA cohort, higher Child-Pugh class, higher TNM tumor burden, and increased visceral fat density were associated with increased risk of post-TACE mortality (Table 3). Each percentile increase in visceral fat density corresponded to $1 \%$ increase in the hazard for mortality (hazard ratio, $1.01 ; 95 \%$ confidence interval, 1.00 to 1.03$)$. Condi- tional inference trees predicting post-TACE mortality confirmed that visceral fat density had the ability to risk stratify these patients (S3 Fig.). The optimal threshold identified is the 56th percentile of visceral fat density, with a higher density resulting in a higher mortality risk. In the AAVA and the UMHS cohorts; $32(43 \%)$ and $54(59 \%)$ patients fell into the high-risk category respectively. The Kaplan-Meier survival curves stratified by this fat density threshold showed a statistically significant difference in 1-year survival in both cohorts; $39 \%$ vs. $78 \%(\mathrm{p}<0.001)$ and $44 \%$ vs. $72 \%(\mathrm{p}<0.001)$ for the AAVA and the UMHS cohorts respectively (Fig. 1A and B). 
A
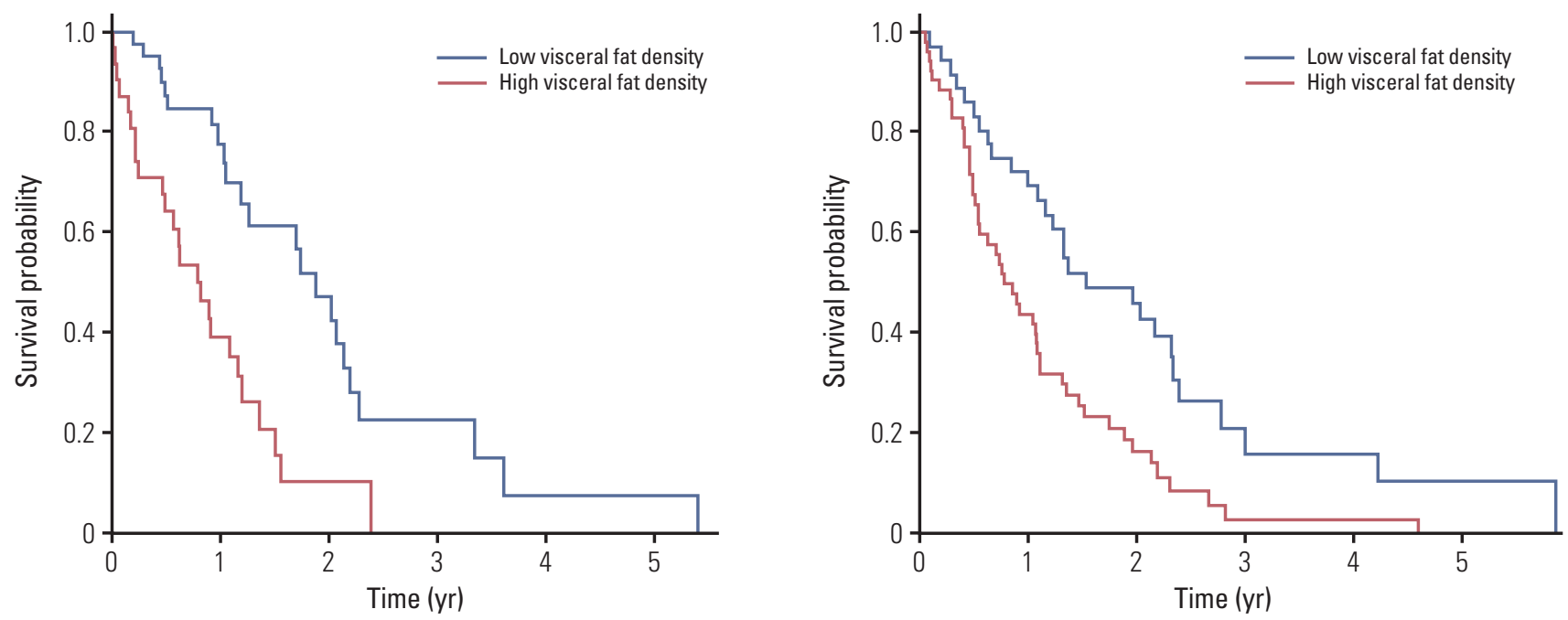

Fig. 1. Kaplan-Meier survival of the derivation cohort (A) and the validation cohort (B) stratified by visceral fat density.

\section{Secondary outcomes}

A total of $27 \%$ patients in the AAVA cohort had decompensation after TACE, with a median time from TACE to decompensation of 5 days (the first and third quartiles are 2.75 and 14 days, respectively). As expected, patients with decompensation after TACE had significantly worse 1-year survival than those without decompensation (27\% vs. $74 \%$, $\mathrm{p}<0.001)$. In univariate analysis, patients with hepatic decompensation after TACE had significantly higher visceral fat density (median, 76; interquartile range [IQR], 61 to 90) than those without decompensation (median, 34; IQR, 18 to 60) $(\mathrm{p}<0.001)$. In a multivariate logistic regression model with similar forward/backward selection as Cox regression, visceral fat density remained significantly associated with development of hepatic decompensation ( $<$ 0.001) (S4 Table).

Patients with ascites at time of TACE had significantly higher visceral fat density when compared to those without a history of ascites (84 vs. 36, p < 0.001). In our sub-analysis of patients in both cohorts who were TACE eligible according to BCLC criteria $(n=109)$, there were 57 patients above and 52 patients below the 56th percentile. Patients in the higher density group had significantly worse 1-year survival ( $31 \%$ vs $67 \%$, p < 0.001.) We next performed an exploratory subgroup analyses in those with Child A cirrhosis and those with Child-Pugh scores 5-7 to evaluate if visceral fat density could be a marker of early portal hypertension. Among Child-Pugh class A patients from both cohorts $(n=97)$, the visceral fat density cutoff of 56 was significantly associated with 1-year survival (29\% vs. 80\%, p < 0.001) (Fig. 2). Simi-

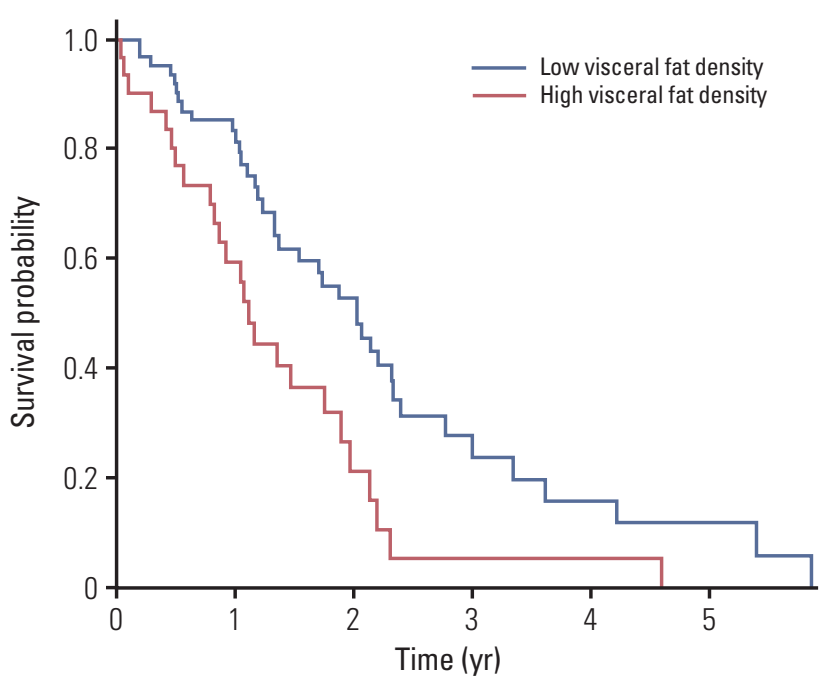

Fig. 2. Kaplan-Meier survival curve of all Child-Pugh class A patients from derivation and validation cohort stratified by visceral fat density.

larly, in patients with Child-Pugh scores of 5-7 ( $n=120)$, the visceral fat density cutoff of $56 \%$ was significantly associated with 1-year survival ( $31 \%$ vs. $73 \%, \mathrm{p}<0.001)$. By contrast, in the subset of patients with Child-Pugh score 8-12, visceral fat density was not predictive of outcome as this feature is unlikely to differentiate patients with more advanced liver disease. 


\section{Discussion}

There are limited clinical tools and biomarkers for predicting outcomes for patients undergoing locoregional therapies for HCC. We have shown that use of analytic morphomic measurements, specifically visceral fat density at T11, can objectively predict the risk of hepatic decompensation and survival in patients undergoing TACE. In the multivariate analysis, each percentage change in the visceral fat density reflected a $1 \%$ change in the hazard survival probability. The prognostic value of visceral fat density cutoff derived from the AAVA cohort was confirmed in an external validation cohort of patients from UMHS, an academic medical center with a demographically dissimilar population from the AAVA cohort.

Our secondary analyses suggest that visceral fat density may be associated with portal hypertension and can potentially predict development of hepatic decompensation after TACE. Importantly, visceral fat density is predictive of survival even in a combined cohort of well compensated ChildPugh class A patients, who traditionally are thought to do well after TACE. We hypothesize that visceral fat density could serve as a marker of early portal hypertension that is present prior to the development of ascites and other overt clinical manifestations of portal hypertension. Higher visceral fat density may correlate with an increase with shunting of portal blood flow or local edema in the interstitial tissue. As fat is less dense than water, an increase in water within the fat would increase overall measurement of visceral fat density. This may explain why patients with higher visceral fat density values predicted decompensation and suggests that this morphomics measure may be an early noninvasive marker for portal hypertension.

A priori predictors of outcome for patients undergoing TACE are largely limited to traditional predictors of decompensation and overall survival including liver function and tumor burden, with patients with poorer liver function and higher tumor burden experiencing worse outcome after TACE [4,22-24]. Analytic morphomics could be a more sensitive prognostic marker than traditional clinical variables, and may be particularly useful in patients with intact liver function.

The limitations of our study include inherent biases associated with retrospective study design. Although analytic morphomics is versatile, at the present time we can only perform image analysis on CT data files and for patients who only had an MRI, we were unable to include them in the study. However, patients with HCC undergo a CT scan of their thorax for staging purposes, thus morphomic information at the T11 level is typically available for all patients with HCC [16]. Another limitation is variation in TACE technique (doxorubicin-eluting bead TACE vs. conventional TACE) given long study duration, however studies have shown equivalent efficacy in the treatment of HCC $[25,26]$. Additionally, we collected morphomics data at baseline before first TACE but locoregional therapies can be applied after the first TACE and may impact 1-year survival. Other unmeasured or poorly measured confounders for survival not were captured given retrospective nature of this analysis. We also included patients who fall outside of BCLC treatment guidelines for TACE (BCLC C and D), but real world analyses have shown poor adherence to BCLC guidelines in clinical practice with lack of impact on outcomes [27]. Finally, our cohorts are relatively small, limiting our power and ability to conduct more nuanced sub-analyses. Future steps would include validation in larger cohorts of patients undergoing TACE and potential development of a clinical prediction model, which incorporates clinical factors with analytic morphomic characteristics.

In conclusion, we have shown that an analytic morphomic parameter, namely visceral fat density, is a prognostic factor for survival after TACE in both a derivation and external validation cohort of patients with HCC. Visceral fat density could be a surrogate for early portal hypertension and can be used as an objective risk stratification method for patients with HCC undergoing TACE. While further validation is required, visceral fat density may play a role as a radiographic biomarker in clinical risk prediction for patients undergoing TACE.

\section{Electronic Supplementary Material}

Supplementary materials are available at Cancer Research and Treatment website (http://www.e-crt.org).

\section{Conflicts of Interest}

Conflict of interest relevant to this article was not reported.

\section{Acknowledgments}

P.Z. was partially supported by the National Institute of Diabetes and Digestive and Kidney Diseases 1K01DK106296. 


\section{References}

1. Petrick JL, Kelly SP, Altekruse SF, McGlynn KA, Rosenberg PS. Future of hepatocellular carcinoma incidence in the United States forecast through 2030. J Clin Oncol. 2016;34: 1787-94.

2. Llovet JM, Bru C, Bruix J. Prognosis of hepatocellular carcinoma: the BCLC staging classification. Semin Liver Dis. 1999;19:329-38.

3. Barman PM, Su GL. Limitations of the Barcelona Clinic Liver Cancer Staging System with a focus on transarterial chemoembolization as a key modality for treatment of hepatocellular carcinoma. Clin Liver Dis. 2016;7:32-5.

4. Barman PM, Sharma P, Krishnamurthy V, Willatt J, McCurdy $\mathrm{H}$, Moseley RH, et al. Predictors of mortality in patients with hepatocellular carcinoma undergoing transarterial chemoembolization. Dig Dis Sci. 2014;59:2821-5.

5. Ryerson AB, Eheman CR, Altekruse SF, Ward JW, Jemal A, Sherman RL, et al. Annual report to the nation on the status of cancer, 1975-2012, featuring the increasing incidence of liver cancer. Cancer. 2016;122:1312-37.

6. Jeon HJ, Shim EJ, Shin YW, Oh DY, Im SA, Heo DS, et al. Discrepancies in performance status scores as determined by cancer patients and oncologists: are they influenced by depression? Gen Hosp Psychiatry. 2007;29:555-61.

7. Kelly CM, Shahrokni A. Moving beyond Karnofsky and ECOG performance status assessments with new technologies. J Oncol. 2016;2016:6186543.

8. Englesbe MJ, Lee JS, He K, Fan L, Schaubel DE, Sheetz KH, et al. Analytic morphomics, core muscle size, and surgical outcomes. Ann Surg. 2012;256:255-61.

9. Krishnamurthy V, Zhang P, Ethiraj S, Enchakalody B, Waljee AK, Wang L, et al. Use of analytic morphomics of liver, spleen, and body composition to identify patients at risk for cirrhosis. Clin Gastroenterol Hepatol. 2015;13:360-8.

10. Sharma P, Parikh ND, Yu J, Barman P, Derstine BA, Sonnenday $\mathrm{CJ}$, et al. Bone mineral density predicts posttransplant survival among hepatocellular carcinoma liver transplant recipients. Liver Transpl. 2016;22:1092-8.

11. Stidham RW, Waljee AK, Day NM, Bergmans CL, Zahn KM, Higgins PD, et al. Body fat composition assessment using analytic morphomics predicts infectious complications after bowel resection in Crohn's disease. Inflamm Bowel Dis. 2015;21:1306-13.

12. Singal AG, Zhang P, Waljee AK, Ananthakrishnan L, Parikh ND, Sharma $P$, et al. Body composition features predict overall survival in patients with hepatocellular carcinoma. Clin Transl Gastroenterol. 2016;7:e172.

13. Yopp AC, Mansour JC, Beg MS, Arenas J, Trimmer C, Reddick $\mathrm{M}$, et al. Establishment of a multidisciplinary hepatocellular carcinoma clinic is associated with improved clinical outcome. Ann Surg Oncol. 2014;21:1287-95.

14. Patel N, Yopp AC, Singal AG. Diagnostic delays are common among patients wtih hepatocellular carcinoma. J Natl Compr
Canc Netw. 2015;13:543-9.

15. Singal AG, Yopp AC, Gupta S, Skinner CS, Halm EA, Okolo $\mathrm{E}$, et al. Failure rates in the hepatocellular carcinoma surveillance process. Cancer Prev Res (Phila). 2012;5:1124-30.

16. Bruix J, Sherman M; American Association for the Study of Liver Diseases. Management of hepatocellular carcinoma: an update. Hepatology. 2011;53:1020-2.

17. Huhdanpaa H, Douville C, Baum K, Krishnamurthy VN, Holcombe S, Enchakalody B, et al. Development of a quantitative method for the diagnosis of cirrhosis. Scand J Gastroenterol. 2011;46:1468-77.

18. Zhang P, Parenteau C, Wang L, Holcombe S, Kohoyda-Inglis C, Sullivan J, et al. Prediction of thoracic injury severity in frontal impacts by selected anatomical morphomic variables through model-averaged logistic regression approach. Accid Anal Prev. 2013;60:172-80.

19. Harbaugh CM, Terjimanian MN, Lee JS, Alawieh AZ, Kowalsky DB, Tishberg LM, et al. Abdominal aortic calcification and surgical outcomes in patients with no known cardiovascular risk factors. Ann Surg. 2013;257:774-81.

20. Peterson MD, McGrath R, Zhang P, Markides KS, Al Snih S, Wong R. Muscle weakness is associated with diabetes in older Mexicans: the Mexican health and aging study. J Am Med Dir Assoc. 2016;17:933-8.

21. Clark TW. Complications of hepatic chemoembolization. Semin Intervent Radiol. 2006;23:119-25.

22. Kadalayil L, Benini R, Pallan L, O'Beirne J, Marelli L, Yu D, et al. A simple prognostic scoring system for patients receiving transarterial embolisation for hepatocellular cancer. Ann Oncol. 2013;24:2565-70.

23. Kohla MA, Abu Zeid MI, Al-Warraky M, Taha H, Gish RG. Predictors of hepatic decompensation after TACE for hepatocellular carcinoma. BMJ Open Gastroenterol. 2015;2:e000032.

24. Kirstein MM, Schweitzer N, Ay N, Boeck C, Lappas K, Hinrichs JB, et al. Experience from a real-life cohort: outcome of 606 patients with hepatocellular carcinoma following transarterial chemoembolization. Scand J Gastroenterol. 2017;52: 116-24.

25. Frenette CT, Osorio RC, Stark J, Fok B, Boktour MR, Guy J, et al. Conventional TACE and drug-eluting bead TACE as locoregional therapy before orthotopic liver transplantation: comparison of explant pathologic response. Transplantation. 2014;98:781-7.

26. Hui Y, Ruihua T, Jing L, Yaxiong L, Ji L, Linjie Y, et al. Metaanalysis of doxorubicin-eluting beads via transcatheter arterial chemoembolization in the treatment of unresectable hepatocellular carcinoma. Hepatogastroenterology. 2015;62:1002-6.

27. Mathew J, Slipak S, Kotru A, Blansfield J, Woll N, Shabahang $\mathrm{M}$. Adherence to the BCLC guidelines and impact on overall survival. J Clin Oncol. 2014;32(3 Suppl):Abstr 345. 